\title{
Caracterização da morbidade de crianças/adolescentes com cuidados contínuos e complexos internados em terapia intensiva pediátrica
}

Characterization of morbidity in children/adolescents with continuous and complex care admitted

to pediatric intensive care

Caracterización de la morbilidad en niños / adolescentes con cuidados continuos y complejos ingresados en cuidados intensivos pediátricos

\section{Resumo}

Objetivo: analisar o perfil da morbidade de crianças/adolescentes com cuidados contínuos e complexos egressos de uma Unidade de Terapia Intensiva Pediátrica e os fatores associados à necessidade de cuidados contínuos e complexos. Método: estudo quantitativo, retrospectivo, do tipo documental, desenvolvido a partir da análise estatística descritiva e analítica dos prontuários de crianças/adolescentes com cuidados contínuos e complexos de uma Unidade de Terapia Intensiva Pediátrica do estado do Rio Grande do Sul. A análise dos dados foi realizada por meio de estatística descritiva e analítica. Resultados: doenças do aparelho respiratório foram as principais causas de internação nessa unidade. Predominaram crianças de até um ano de idade, de etnia branca e do sexo masculino. História prévia de morbidades, uso de medicação contínua, ventilação mecânica e tecnologia em saúde mostraram-se associadas à necessidade de cuidados contínuos e complexos. Conclusão: existem condições que predispõem a necessidade de cuidados contínuos e complexos, implicando na sistematização de ações direcionadas à diminuição da sua necessidade e do número de reinternações dessa clientela.

Palavras-chave: Indicadores de morbidade; Unidades de Terapia Intensiva Pediátrica; Criança; Adolescente; Enfermagem.

\footnotetext{
Abstract

Objective: to analyze the morbidity profile of children/adolescents with continuous and complex care discharged from a Pediatric Intensive Care Unit and the factors associated with the need for continuous and complex care. Method: quantitative, retrospective, documentary study, developed from the descriptive and analytical statistical analysis of medical records of children/adolescents with continuous and complex care in a Pediatric Intensive Care Unit in the state of Rio Grande do Sul. Data analysis was performed using descriptive and analytical statistics. Results: diseases of the respiratory system were the main causes of admission to this unit. Children aged up to one year, white and male predominated. Previous history of morbidities, use of continuous medication, mechanical ventilation and health
} 
technology were associated with the need for continuous and complex care. Conclusion: there are conditions that predispose the need for continuous and complex care, implying the systematization of actions aimed at reducing its need and the number of readmissions for this clientele.

Keywords: Morbidity indicators; Intensive Care Units, Pediatric; Child; Adolescent; Nursing.

\section{Resumen}

Objetivo: analizar el perfil de morbilidad de niños / adolescentes con cuidados continuos y complejos dados de alta de una Unidad de Cuidados Intensivos Pediátricos y los factores asociados a la necesidad de cuidados continuos y complejos. Método: estudio cuantitativo, retrospectivo, documental, desarrollado a partir del análisis estadístico descriptivo y analítico de historias clínicas de niños / adolescentes con atención continua y compleja en una Unidad de Cuidados Intensivos Pediátricos en el estado de Rio Grande do Sul. El análisis de datos se realizó mediante métodos descriptivos y estadísticas analíticas. Resultados: las enfermedades del aparato respiratorio fueron las principales causas de ingreso a esta unidad. Predominaron los niños de hasta un año, blancos y varones. La historia previa de morbilidad, el uso de medicación continua, la ventilación mecánica y la tecnología sanitaria se asociaron a la necesidad de cuidados continuos y complejos. Conclusión: existen condiciones que predisponen la necesidad de una atención continua y compleja, lo que implica la sistematización de acciones encaminadas a reducir su necesidad y el número de reingresos de esta clientela.

Palabras clave: Indicadores de morbilidad; Unidades de Cuidado Intensivo Pediátrico; Niño; Adolescente; Enfermería.

\section{Introdução}

As transformações nas esferas sociais, econômicas e políticas que se sucederam ao longo dos anos proporcionaram uma nova percepção da criança no mundo e, assim, o interesse social pela mesma. Isso pode ser considerado um reflexo da acelerada evolução medicamentosa, equipamentos e recursos humanos direcionados à possibilidade de viabilizar a vida para crianças portadoras de doenças crônicas (Cruz et al., 2017). Com essas transformações, na década de 30 do século XX, foram estruturadas políticas públicas voltadas à maternidade, infância e adolescência, essencialmente, centradas na redução dos óbitos infantis e maternos.

A partir disso, a criação de Unidades de Terapia Intensiva Pediátricas (UTIP) contribuíram significativamente para a redução dos óbitos infantis. As Unidades de Terapia Intensiva (UTI), segundo a Resolução nº 7, de 24 de fevereiro de 2010 , são locais indicados às internações de pacientes que estão em estado crítico de saúde, que têm a necessidade de atenção contínua de profissionais especializados, equipamentos e tecnologias para diagnóstico e tratamento de saúde (Brasil, 2010).

Tais avanços nas políticas públicas e nas tecnologias na área da saúde propiciaram mudanças no âmbito do perfil epidemiológico da infância em nível mundial, com diminuição das taxas de mortalidade infantil e aumento das doenças crônicas. Nesse contexto, surge um grupo de crianças que, inicialmente, nos Estados Unidos, foram denominadas de Children With Special Health Care Needs (CSHCN) (Mcpherson et al., 1998). No Brasil, elas são conhecidas como Crianças com Necessidades Especiais de Saúde (CRIANES), que correspondem ao grupo de crianças e adolescentes com idades entre 0 e 18 anos (Silveira \& Neves, 2019). Estas são portadoras de condições crônicas de vida, sejam elas de cunho comportamental, emocional, físico ou desenvolvimental, de forma a demandar atendimentos especializados nos serviços de saúde por equipe multiprofissional devido à sua fragilidade clínica (Arrué et al., 2016; Góes \& Cabral, 2017).

Esse grupo de crianças compõe uma clientela em ascensão, caracterizando um desafio para os profissionais de saúde. Tal clientela requer demandas de cuidados específicos, as quais podem ser classificadas como de desenvolvimento, tecnológicas, medicamentosas e/ou habituais modificadas e mistas (Neves \& Cabral, 2009). Inserido no grupo das CRIANES, tem-se o subgrupo das crianças/adolescentes medicamente frágeis, com cuidados médicos complexos, dependentes de tecnologia e crianças com condições crônicas complexas (Cohen et al., 2011; Simon et al., 2010). Estas apresentam problemas de saúde com maior complexidade e maiores demandas de cuidados de saúde, bem como condições crônicas de saúde ao longo da sua vida consideradas graves e/ou associadas à fragilidade clínica, limitação funcional, uso de recursos na saúde de alta complexidade e hospitalizações e/ou demandas de múltiplas especialidades médicas (Cohen et al., 2011; Sulino et al., 2021).

As CRIANES, quando recebem alta e retornam para os seus domicílios, demandam de cuidados contínuos e de natureza 
complexa, surgindo como desafios aos seus cuidadores. Essas crianças possuem, por vezes, pouca visibilidade nos serviços de atenção primária à saúde (APS) e seu acesso é permeado por obstáculos, com ênfase para a lentidão no processo de definição do diagnóstico e encaminhamento para serviço especializado, despreparo no atendimento oferecido em relação as suas necessidades, além de barreiras logísticas de acessibilidade (Neves et al., 2019; Sulino et al., 2021). Percebem-se lacunas nas políticas públicas específicas direcionadas à atuação de profissionais da APS, pois estas unidades são vistas, muitas vezes, como pouco resolutivas, refletindo na fragmentação do cuidado, fragilização de vínculos entre profissionais, famílias e instituições de saúde, além de transmissão precária de orientações para familiares sobre cuidados domiciliares (Silva et al., 2017; Neves et al., 2019).

Nesse sentido, existe a necessidade de o profissional de enfermagem atuar como mediador, para que possam ser elaboradas práticas de cuidado, tendo sempre como base os conhecimentos fundamentais da enfermagem associados à educação em saúde, e uma vez que essa estratégia leve em consideração o conhecimento dos familiares, que são, na maioria dos casos, os responsáveis pelo cuidado dessa criança no domicílio. Assim, quando a educação em saúde acontece de forma efetiva, é possível atuar na redução das taxas de reinternações (Okido et al., 2016).

Diante do exposto, questionou-se: qual o perfil da morbidade de crianças/adolescentes com cuidados contínuos e complexos egressos de uma UTIP e os fatores associados à necessidade desses cuidados contínuos e complexos? Para responder a esse questionamento, objetivou-se analisar o perfil da morbidade de crianças/adolescentes com cuidados contínuos e complexos egressos de uma UTIP e os fatores associados à necessidade de cuidados contínuos e complexos.

Justifica-se a necessidade da realização desse estudo para conhecer e demarcar a existência dessas crianças/adolescentes na terapia intensiva pediátrica, ao longo do tempo, e suas demandas de cuidados contínuos e complexos que repercutem no planejamento da alta hospitalar para os cuidados domiciliares que serão desenvolvidos por seus familiares.

\section{Metodologia}

Trata-se de um estudo quantitativo, do tipo documental e retrospectivo (Polit \& Beck, 2011) desenvolvido a partir do banco de dados do projeto matricial intitulado "Caracterização da morbimortalidade de crianças/adolescentes egressos de uma Unidade de Terapia Intensiva Pediátrica". O projeto matricial tem como objetivo geral analisar a morbimortalidade de crianças e adolescentes egressos de uma UTIP nos anos de 1995, 1997, 2005, 2007, 2015 e 2017. Considerando-se que a inauguração da UTIP foi em 1995, sendo este o primeiro ano da pesquisa, os anos seguintes foram estratificados por biênios 1995/1997; 2005/2007 e 2015/2017, a fim de acompanhar a evolução da morbimortalidade de uma forma representativa no período de 1995 a 2017.

Os dados foram coletados de julho de 2018 a maio de 2019 por uma equipe de coletadores, previamente capacitados. Em um primeiro momento foi feito o levantamento das crianças e adolescentes que estiveram internados nos anos de $1995 \mathrm{e}$ 1997 a partir do livro de registros da unidade. Para os demais anos foi utilizada a planilha disponibilizada pelo setor de estatística do hospital. Desta forma, estas informações serviram de guia para a solicitação dos prontuários no setor de arquivo do hospital.

O cenário do estudo foi uma UTIP de um hospital público da região central do Rio Grande do Sul. Foram acessados prontuários de crianças e adolescentes que estiveram hospitalizados na referida unidade nos anos supracitados, considerando criança na faixa etária até doze anos de idade incompletos e adolescente, de doze a dezoito anos, conforme consta no Estatuto da Criança e do Adolescente (Brasil, 1990). Foram excluídos os prontuários em que não constava a nota de alta ou de óbito, que fosse de internação oriunda de recuperação de exames ou pequenas cirurgias, como inserção de cateter venoso central, e prontuários que não foram disponibilizados após cinco tentativas. Após a aplicação dos critérios de exclusão, restaram 111 prontuários no ano de 1995, 189 em 1997, 68 em 2005, 116 em 2007, 175 em 2015 e 168 em 2017, totalizando 827 prontuários.

Para a coleta dos dados, utilizou-se um instrumento elaborado para o projeto matricial, previamente testado, organizado em cinco blocos, sendo eles: Bloco A - Identificação; Bloco B - Perfil demográfico; Bloco C - Perfil de nascimento; Bloco D 
- Perfil clínico; e Bloco E - Perfil da alta hospitalar. Destaca-se que, para este estudo, foram utilizados os dados dos Blocos B e D. O Bloco B está constituído pelas variáveis: data de nascimento, sexo, etnia, naturalidade e procedência. Já, o Bloco D, pelas variáveis: data da internação, motivo de internação, hipótese diagnóstica, história diagnóstica, proveniente de qual unidade, necessidade de reanimação e manobras realizadas, uso de ventilação mecânica, necessidade e tipo de procedimento cirúrgico, número de reinternações, ocorrência, data e motivo do óbito ou data da alta da UTIP.

Para os resultados apresentados neste artigo foram selecionadas todas as crianças e adolescentes com cuidados contínuos e complexos do projeto matricial. Destaca-se que foram consideradas com cuidados contínuos e complexos, aquelas crianças e adolescentes que apresentassem pelo menos duas demandas de cuidado, dentre as quais: habitual modificado, medicamentoso, tecnológico ou de desenvolvimento. Assim, têm-se respectivamente nos anos de 1995, 1997, 2005, 2007, 2015 e 2017, um total de 23, 17, 9, 49, 64 e 36 crianças e adolescentes com cuidados contínuos e complexos, totalizando 198 prontuários.

Dividiu-se as crianças e adolescentes por classificação de faixa etária: lactância até 1 ano de idade; primeira infância de 1 a 6 anos; infância intermediária de 6 a 12 anos incompletos; e infância tardia (adolescência) de 12 a 18 anos completos (Hockenberry \& Wilson, 2014). As cidades que compõem as variáveis de naturalidade e procedência foram divididas pelas macrorregiões de saúde do estado do Rio Grande do Sul, quais sejam: norte, sul, centro-oeste, missioneira, metropolitana e vales. Os diagnósticos, sinais e sintomas que formaram as variáveis de história diagnóstica foram categorizados de acordo com a Décima Classificação Internacional de Doenças (CID-10).

Os dados foram digitados e organizados no programa Epi-info® (versão 7.2.3.1), sob dupla digitação independente. Após verificadas e corrigidas as inconsistências, a análise dos dados foi feita no programa estatístico R (versão 3.6.1). As variáveis qualitativas foram analisadas por meio das frequências absoluta e relativa. A análise bivariada foi realizada testando a associação entre a necessidade de cuidado contínuo e complexo (variável dependente) e as variáveis demográficas e clínicas das crianças e adolescentes, mediante o Teste do Qui-quadrado de Pearson, o Teste do Qui-quadrado com correção de continuidade de Yates (para tabelas 2x2 com frequência esperada entre 5 e 10) ou o Teste Exato de Fisher (para tabelas $2 \times 2$ com frequência esperada inferior a 5). Considerou-se com nível de significância quando $\mathrm{p}<0,05$.

O projeto matricial foi desenvolvido de acordo com a Resolução 466/2012 do Conselho Nacional de Saúde e foi aprovado pelo Comitê de Ética em Pesquisa da Universidade Federal de Santa Maria, sob parecer de número: 2.711 .094 e Certificado de Apresentação para Apreciação Ética: 89344518.1.0000.5346.

\section{Resultados}

A partir da análise dos resultados do estudo, observou que crianças e adolescentes com cuidados contínuos e complexos representaram 20,7\% (N=23) do total de internações na UTIP no ano de 1995; 9\% (N=17) em 1997; 13,2\% (N=9) em 2005; 42,2\% (N=49) em 2007; 36,5\% ( $=64)$ em 2015; e 21,4\% (N=36) em 2017. Identificou-se também que os participantes, em sua maioria, eram do sexo masculino (1995=60,9\%; 1997=58,8\%; 2007=63,3\%; 2015=57,8\%; 2017=52,8\%), de etnia branca $(1995=78,3 \% ; 1997=76,5 \% ; 2005=55,6 \% ; 2007=67,4 \% ; 2015=85,9 \% ; 2017=72,2 \%)$. Com relação à faixa etária, o maior percentual possuía entre zero e um ano de idade (1995=60,9\%; 1997=70,6\%; 2005=44,4\%; 2007=40,8\%; 2015=40,6\%; 2017=38,9\%). Os participantes eram procedentes da macrorregião de saúde centro-oeste do estado do Rio Grande do Sul $(1995=78,2 \% ; 1997=70,6 \% ; 2005=88,9 \% ; 2007=91,8 \% ; 2015=81,2 \% ; 2017=77,8 \%)$.

Na Tabela 1 apresentam-se as hipóteses diagnósticas das crianças e adolescentes com cuidados contínuos e complexos, agrupadas de acordo com o CID-10. 
Tabela 1 - Hipóteses diagnósticas, agrupadas pelo CID-10, das crianças e adolescentes com cuidados contínuos e complexos internados na Unidade de Terapia Intensiva Pediátrica nos anos de 1995, 1997, 2005, 2007, 2015 e 2017. Santa Maria, RS, Brasil, 2021.

\begin{tabular}{|c|c|c|c|c|c|c|}
\hline \multirow{2}{*}{ Capítulos do CID-10 } & 1995 & 1997 & 2005 & 2007 & 2015 & 2017 \\
\hline & $\mathbf{N}(\%)$ & $\mathbf{N}(\%)$ & $\mathbf{N}(\%)$ & $\mathbf{N}(\%)$ & $\mathbf{N}(\%)$ & $\mathbf{N}(\%)$ \\
\hline $\begin{array}{l}\text { Algumas doenças infecciosas e } \\
\text { parasitárias }\end{array}$ & $5(12,8)$ & $1(4,8)$ & $2(18,2)$ & $10(15,6)$ & $8(9,1)$ & $8(16,0)$ \\
\hline Neoplasmas (tumores) & - & - & - & $1(1,6)$ & $4(4,5)$ & - \\
\hline $\begin{array}{l}\text { Doenças do sangue e dos órgãos } \\
\text { hematopoéticos e alguns } \\
\text { transtornos imunitários }\end{array}$ & $2(5,1)$ & - & - & $2(3,1)$ & $2(2,2)$ & $2(4,0)$ \\
\hline $\begin{array}{l}\text { Doenças endócrinas, } \\
\text { nutricionais e metabólicas }\end{array}$ & $1(2,6)$ & $1(4,8)$ & $1(9,1)$ & $8(12,5)$ & $7(7,9)$ & $4(8,0)$ \\
\hline Doenças do sistema nervoso & $11(28,2)$ & $3(14,2)$ & $2(18,2)$ & $6(9,4)$ & $10(11,2)$ & $6(12,0)$ \\
\hline $\begin{array}{lll}\begin{array}{l}\text { Doenças } \\
\text { circulatório }\end{array} & \text { do } & \text { aparelho } \\
\end{array}$ & $3(7,7)$ & - & $1(9,1)$ & $5(7,8)$ & $4(4,5)$ & - \\
\hline Doença do aparelho respiratório & $13(33,3)$ & $12(57,0)$ & $2(18,2)$ & $19(29,7)$ & $22(24,7)$ & $15(30,0)$ \\
\hline Doenças do aparelho digestivo & - & $1(4,8)$ & - & $2(3,1)$ & $7(7,9)$ & $3(6,0)$ \\
\hline $\begin{array}{lrrr}\begin{array}{l}\text { Doenças } \\
\text { osteomuscular }\end{array} & \text { e } & \text { do } & \text { tecido } \\
\text { conjuntivo } & & & \end{array}$ & - & - & - & - & $1(1,1)$ & - \\
\hline $\begin{array}{l}\text { Doenças do aparelho } \\
\text { geniturinário }\end{array}$ & $1(2,6)$ & - & - & $1(1,6)$ & $2(2,2)$ & $2(4,0)$ \\
\hline $\begin{array}{l}\text { Algumas afecções originadas no } \\
\text { período perinatal }\end{array}$ & - & $1(4,8)$ & - & - & $3(3,4)$ & $2(4,0)$ \\
\hline $\begin{array}{l}\text { Malformações } \\
\text { deformidades } \\
\text { cromossômicas }\end{array} \quad \begin{array}{r}\text { congênitas, } \\
\text { anomalias }\end{array}$ & $3(7,7)$ & $1(4,8)$ & $1(9,1)$ & $3(4,7)$ & $7(7,9)$ & $6(12,0)$ \\
\hline $\begin{array}{l}\text { Sintomas, sinais e achados } \\
\text { anormais de exames clínicos e } \\
\text { de laboratório, não classificados } \\
\text { em outra parte }\end{array}$ & - & - & - & - & $1(1,1)$ & - \\
\hline $\begin{array}{l}\text { Lesões, envenenamentos e } \\
\text { algumas outras consequências } \\
\text { de causas externas }\end{array}$ & - & $1(4,8)$ & $2(18,2)$ & $7(10,9)$ & $10(11,2)$ & $2(4,0)$ \\
\hline $\begin{array}{l}\text { Fatores que influenciam o estado } \\
\text { de saúde e o contato com os } \\
\text { serviços de saúde }\end{array}$ & - & - & - & - & $1(1,1)$ & - \\
\hline Total* & 39 & 21 & 11 & 64 & 89 & 50 \\
\hline
\end{tabular}

*Algumas crianças e adolescentes possuíam mais de uma hipótese diagnóstica, por esse motivo essa variável apresenta um $\mathrm{N}$ diferente do total de crianças e adolescentes do estudo. Fonte: Elaborado pelos autores (2021).

Observa-se que as doenças do aparelho respiratório (1995=33,3\%; 1997=57\%; 2005=18,2\%; 2007=29,7\%; $2015=24,7 \% ; 2017=30 \%$ ) constituíram-se na principal hipótese diagnóstica apresentada pelas crianças e adolescentes com cuidados contínuos e complexos. Outras que aparecem como destaque são doenças do sistema nervoso (1995=28,2\%; $1997=14,2 \% ; 2005=18,2 \% ; 2015=11,2 \%)$, doenças infecciosas e parasitárias $(2005=18,2 \% ; 2007=15,6 \% ; 2017=16 \%)$ e lesões, envenenamentos e algumas outras consequências de causas externas $(2005=18,2 \% ; 2015=11,2 \%)$.

A Tabela 2 apresenta a associação da necessidade de cuidado contínuo e complexo com as variáveis nos anos de 1995 e 1997. 
Tabela 2 - Necessidade de cuidados contínuos e complexos segundo variáveis demográficas e clínicas das crianças e adolescentes internadas na Unidade de Terapia Intensiva Pediátrica nos anos de 1995 e 1997. Santa Maria, RS, Brasil, 2021.

\begin{tabular}{|c|c|c|c|c|c|c|c|c|}
\hline \multirow{3}{*}{ Variáveis } & \multicolumn{8}{|c|}{ Necessidade de cuidados contínuos e complexos } \\
\hline & \multicolumn{4}{|c|}{1995} & \multicolumn{4}{|c|}{1997} \\
\hline & $\frac{\text { Sim }}{\mathbf{N}(\%)}$ & $\begin{array}{c}\text { Não } \\
\text { N(\%) }\end{array}$ & $\begin{array}{c}\text { Total } \\
\mathbf{N}(\%)\end{array}$ & p-value & $\begin{array}{c}\text { Sim } \\
\mathbf{N}(\%)\end{array}$ & $\begin{array}{c}\text { Não } \\
\text { N (\%) }\end{array}$ & $\begin{array}{c}\text { Total } \\
\mathbf{N}(\%)\end{array}$ & p-value \\
\hline \multicolumn{9}{|l|}{ Sexo } \\
\hline Feminino & $9(11,0)$ & $27(32,9)$ & $36(43,9)$ & \multirow{2}{*}{$0,587 *$} & $7(4,6)$ & $60(39,2)$ & $67(43,8)$ & \multirow{2}{*}{$1,000 * *$} \\
\hline Masculino & $14(17,1)$ & $32(39,0)$ & $46(56,1)$ & & $10(6,5)$ & $76(49,7)$ & $86(56,2)$ & \\
\hline \multicolumn{9}{|l|}{ Faixa etária } \\
\hline$\leq 1$ ano & $14(17,1)$ & $33(40,2)$ & $47(57,3)$ & \multirow{4}{*}{$0,354 *$} & $12(7,8)$ & $62(40,5)$ & $74(48,3)$ & \multirow{4}{*}{$0,235^{*}$} \\
\hline$>1-6$ anos & $9(11,0)$ & $21(25,6)$ & $30(36,6)$ & & $3(2,0)$ & $52(34,0)$ & $55(36,0)$ & \\
\hline$>6-<12$ anos & $0(0,0)$ & $5(6,1)$ & $5(6,1)$ & & $2(1,3)$ & $18(11,8)$ & $20(13,0)$ & \\
\hline$\geq 12$ anos & $0(0,0)$ & $0(0,0)$ & $0(0,0)$ & & $0(0,0)$ & $4(2,6)$ & $4(2,7)$ & \\
\hline \multicolumn{9}{|c|}{$\begin{array}{l}\text { Necessidade de } \\
\text { reanimação } \\
\text { cardiopulmonar }\end{array}$} \\
\hline Sim & $1(1,2)$ & $1(1,2)$ & $2(2,4)$ & \multirow{2}{*}{$0,900 * * *$} & $2(1,3)$ & $5(3,3)$ & $7(4,6)$ & \multirow{2}{*}{$1,000^{* * *}$} \\
\hline Não & $22(26,8)$ & $58(70,7)$ & $80(97,6)$ & & $15(9,8)$ & $131(85,6)$ & $146(95,4)$ & \\
\hline \multicolumn{9}{|c|}{$\begin{array}{l}\text { Uso de ventilação } \\
\text { mecânica }\end{array}$} \\
\hline Sim & $10(12,2)$ & $18(22,0)$ & $28(34,2)$ & \multirow{2}{*}{$0,393^{* *}$} & $7(4,6)$ & $24(15,7)$ & $31(20,3)$ & \multirow{2}{*}{$1,000 * * *$} \\
\hline Não & $13(15,8)$ & $41(50,0)$ & $54(65,8)$ & & $10(6,5)$ & $112(73,2)$ & $122(79,7)$ & \\
\hline \multicolumn{9}{|c|}{$\begin{array}{l}\text { Reinternação } \\
\text { antes da alta } \\
\text { hospitalar }\end{array}$} \\
\hline $\operatorname{Sim}$ & $4(4,9)$ & $6(7,3)$ & $10(12,2)$ & \multirow{2}{*}{$0,900 * * *$} & $2(1,4)$ & $4(2,6)$ & $6(4,0)$ & \multirow{2}{*}{$1,000 * * *$} \\
\hline Não & $19(23,2)$ & $53(64,6)$ & $72(87,8)$ & & $15(9,8)$ & $132(86,3)$ & $147(96,0)$ & \\
\hline \multicolumn{9}{|l|}{$\begin{array}{l}\text { História } \\
\text { diagnóstica }\end{array}$} \\
\hline Sim & $13(15,8)$ & $18(22,0)$ & $31(37,8)$ & \multirow{2}{*}{$0,053 * *$} & $12(7,8)$ & $24(15,7)$ & $36(23,5)$ & \multirow{2}{*}{$1,000 * * *$} \\
\hline Não & $10(12,2)$ & $41(50,0)$ & $51(62,2)$ & & $5(3,3)$ & $112(73,2)$ & $117(76,5)$ & \\
\hline \multicolumn{9}{|c|}{$\begin{array}{l}\text { Uso de tecnologia } \\
\text { em saúde }\end{array}$} \\
\hline Sim & $7(8,5)$ & $2(2,5)$ & $9(11,0)$ & \multirow{2}{*}{$1,000 * * *$} & $7(4,6)$ & $2(1,3)$ & $9(5,9)$ & \multirow{2}{*}{$1,000 * * *$} \\
\hline Não & $16(19,5)$ & $57(69,5)$ & $73(89,0)$ & & $10(6,5)$ & $134(87,6)$ & $144(94,1)$ & \\
\hline \multicolumn{9}{|c|}{$\begin{array}{l}\text { Uso de medicação } \\
\text { contínua }\end{array}$} \\
\hline Sim & $19(23,1)$ & $13(15,9)$ & $32(39,0)$ & \multirow{2}{*}{$0,000 * *$} & $13(8,5)$ & $26(17,0)$ & $39(25,5)$ & \multirow{2}{*}{$1,000 * * *$} \\
\hline Não & $4(4,9)$ & $46(56,1)$ & $50(61,0)$ & & $4(2,6)$ & $110(71,9)$ & $114(74,5)$ & \\
\hline
\end{tabular}

*Teste Qui-quadrado de Pearson. **Teste Qui-quadrado com correção de continuidade de Yates. ***Teste Exato de Fisher. Fonte: Elaborado pelos autores (2021).

A partir dos dados apresentados na Tabela 2, observa-se que as crianças e adolescentes que possuíam cuidados contínuos e complexos eram aquelas com história diagnóstica, ou seja, com doenças pré-existentes e que faziam uso de medicação contínua.

A Tabela 3 apresenta a associação da necessidade de cuidados contínuos e complexos com as variáveis nos anos de 2005 e 2007. 
Tabela 3 - Necessidade de cuidados contínuos e complexos segundo variáveis demográficas e clínicas das crianças e adolescentes internadas na Unidade de Terapia Intensiva Pediátrica nos anos de 2005 e 2007. Santa Maria, RS, Brasil, 2021.

\begin{tabular}{|c|c|c|c|c|c|c|c|c|}
\hline \multirow{3}{*}{ Variáveis } & \multicolumn{8}{|c|}{ Necessidade de cuidados contínuos e complexos } \\
\hline & \multicolumn{4}{|c|}{2005} & \multicolumn{4}{|c|}{2007} \\
\hline & $\begin{array}{c}\text { Sim } \\
\mathbf{N}(\%)\end{array}$ & $\begin{array}{c}\text { Não } \\
\text { N(\%) }\end{array}$ & $\begin{array}{c}\text { Total } \\
\text { N }(\%)\end{array}$ & $p$-value & $\begin{array}{c}\text { Sim } \\
\mathbf{N}(\%)\end{array}$ & $\begin{array}{c}\text { Não } \\
\text { N(\%) }\end{array}$ & $\begin{array}{c}\text { Total } \\
\text { N }(\%)\end{array}$ & p-value \\
\hline \multicolumn{9}{|l|}{ Sexo } \\
\hline Feminino & $5(9,4)$ & $21(39,6)$ & $26(49,1)$ & \multirow{2}{*}{$0,800 * * *$} & $18(17,0)$ & $25(23,6)$ & $43(40,5)$ & \multirow{2}{*}{$0,456^{*}$} \\
\hline Masculino & $4(7,5)$ & $23(43,4)$ & $27(50,9)$ & & $31(29,2)$ & $32(30,2)$ & $63(59,5)$ & \\
\hline \multicolumn{9}{|l|}{ Faixa etária } \\
\hline$\leq 1$ ano & $4(7,5)$ & $17(32,1)$ & $21(39,7)$ & \multirow{4}{*}{$0,055^{*}$} & $20(18,9)$ & $18(17,0)$ & $38(35,8)$ & \multirow{4}{*}{$0,791 *$} \\
\hline$>1-6$ anos & $3(5,7)$ & $15(28,3)$ & $18(34,0)$ & & $14(13,2)$ & $20(18,9)$ & $34(32,1)$ & \\
\hline$>6-<12$ anos & $0(0,0)$ & $11(20,9)$ & $11(20,7)$ & & $12(11,3)$ & $15(14,2)$ & $27(25,5)$ & \\
\hline$\geq 12$ anos & $2(3,8)$ & $1(1,9)$ & $3(5,6)$ & & $3(2,8)$ & $4(3,8)$ & $7(6,6)$ & \\
\hline \multicolumn{9}{|c|}{$\begin{array}{l}\text { Necessidade de } \\
\text { reanimação } \\
\text { cardiopulmonar }\end{array}$} \\
\hline Sim & $1(1,9)$ & $0(0,0)$ & $1(1,9)$ & \multirow{2}{*}{$1,000 * * *$} & $4(3,8)$ & $0(0,0)$ & $4(3,8)$ & \multirow{2}{*}{$1,000 * * *$} \\
\hline Não & $8(15,1)$ & $44(83,0)$ & $52(98,1)$ & & $45(42,5)$ & $57(53,8)$ & $102(96,2)$ & \\
\hline \multicolumn{9}{|c|}{$\begin{array}{l}\text { Uso de ventilação } \\
\text { mecânica }\end{array}$} \\
\hline Sim & $1(1,8)$ & $9(17,0)$ & $13(18,8)$ & \multirow{2}{*}{$0,500 * * *$} & $16(15,1)$ & $15(14,2)$ & $31(29,2)$ & \multirow{2}{*}{$0,475^{*}$} \\
\hline Não & $8(15,1)$ & $35(66,0)$ & $43(81,2)$ & & $33(31,1)$ & $42(39,6)$ & $75(70,8)$ & \\
\hline \multicolumn{9}{|c|}{$\begin{array}{l}\text { Reinternação } \\
\text { antes da alta } \\
\text { hospitalar }\end{array}$} \\
\hline Sim & $0(0,0)$ & $1(1,9)$ & $1(1,9)$ & \multirow{2}{*}{$0,800 * * *$} & $3(2,8)$ & $2(1,9)$ & $5(4,7)$ & \multirow{2}{*}{$0,900 * * *$} \\
\hline Não & $9(17,0)$ & $43(81,1)$ & $52(98,1)$ & & $46(43,4)$ & $55(51,9)$ & $101(95,3)$ & \\
\hline \multicolumn{9}{|l|}{$\begin{array}{l}\text { História } \\
\text { diagnóstica }\end{array}$} \\
\hline $\operatorname{Sim}$ & $3(5,7)$ & $14(26,4)$ & $17(32,1)$ & \multirow{2}{*}{$0,700 * * *$} & $38(35,8)$ & $9(8,5)$ & $46(43,4)$ & \multirow{2}{*}{$0,000 *$} \\
\hline Não & $6(11,3)$ & $30(56,6)$ & $36(67,9)$ & & $11(10,4)$ & $48(45,3)$ & $60(56,6)$ & \\
\hline \multicolumn{9}{|c|}{$\begin{array}{l}\text { Uso de tecnologia } \\
\text { em saúde }\end{array}$} \\
\hline Sim & $3(5,7)$ & $0(0,0)$ & $3(5,7)$ & \multirow{2}{*}{$1,000 * * *$} & $7(6,6)$ & $0(0,0)$ & $7(6,6)$ & \multirow{2}{*}{$1,000 * * *$} \\
\hline Não & $6(11,3)$ & $44(83,0)$ & $50(94,3)$ & & $42(39,6)$ & $57(53,8)$ & $99(93,4)$ & \\
\hline \multicolumn{9}{|c|}{$\begin{array}{l}\text { Uso de medicação } \\
\text { contínua }\end{array}$} \\
\hline Sim & $7(13,2)$ & $22(41,5)$ & $29(54,7)$ & \multirow{2}{*}{$1,000 * * *$} & $31(29,2)$ & $5(4,7)$ & $36(34,0)$ & \multirow{2}{*}{$0,000^{*}$} \\
\hline Não & $2(3,8)$ & $22(41,5)$ & $24(45,3)$ & & $18(17,0)$ & $52(49,1)$ & $70(66,0)$ & \\
\hline
\end{tabular}

*Teste Qui-quadrado de Pearson. ***Teste Exato de Fisher. Fonte: Elaborado pelos autores (2021).

No ano de 2007 evidencia-se, mais uma vez, que crianças com necessidade de cuidados contínuos e complexos apresentam história prévia de morbidades e uso contínuo de medicação. A Tabela 4 apresenta a associação da necessidade de cuidado contínuo e complexo com as variáveis nos anos de 2015 e 2017. 
Tabela 4 - Necessidade de cuidados contínuos e complexos segundo variáveis demográficas e clínicas das crianças e adolescentes internadas na Unidade de Terapia Intensiva Pediátrica nos anos de 2015 e 2017. Santa Maria, RS, Brasil, 2021.

\begin{tabular}{|c|c|c|c|c|c|c|c|c|}
\hline \multirow{4}{*}{ Variáveis } & \multicolumn{8}{|c|}{ Necessidade de cuidados contínuos e complexos } \\
\hline & \multicolumn{4}{|c|}{2015} & \multicolumn{4}{|c|}{2017} \\
\hline & Sim & Não & Total & \multirow{2}{*}{ p-value } & Sim & Não & Total & \multirow{2}{*}{ p-value } \\
\hline & $\mathbf{N}(\%)$ & $\mathbf{N}(\%)$ & $\mathbf{N}(\%)$ & & $\mathbf{N}(\%)$ & $\mathbf{N}(\%)$ & $\mathbf{N}(\%)$ & \\
\hline \multicolumn{9}{|l|}{ Sexo } \\
\hline Feminino & $27(18,5)$ & $32(21,9)$ & $59(40,4)$ & \multirow{2}{*}{$0,699 *$} & $17(11,8)$ & $43(29,9)$ & $60(41,7)$ & \multirow{2}{*}{$0,435^{*}$} \\
\hline Masculino & $37(25,3)$ & $50(34,2)$ & $87(59,6)$ & & $19(13,2)$ & $65(45,1)$ & $84(58,3)$ & \\
\hline \multicolumn{9}{|l|}{ Faixa etária } \\
\hline$\leq 1$ ano & $26(17,8)$ & $40(27,4)$ & $66(45,2)$ & \multirow{4}{*}{$0,479 *$} & $14(9,7)$ & $50(34,7)$ & $64(44,4)$ & \multirow{4}{*}{$0,331 *$} \\
\hline$>1-6$ anos & $19(13,0)$ & $18(12,3)$ & $37(25,3)$ & & $9(6,3)$ & $30(20,8)$ & $39(27,1)$ & \\
\hline$>6-<12$ anos & $14(9,6)$ & $14(9,6)$ & $28(19,2)$ & & $6(4,2)$ & $19(13,2)$ & $25(17,4)$ & \\
\hline$\geq 12$ anos & $5(3,4)$ & $10(6,8)$ & $15(10,3)$ & & $7(4,9)$ & $9(6,3)$ & $16(11,2)$ & \\
\hline \multicolumn{9}{|c|}{$\begin{array}{l}\text { Necessidade de } \\
\text { reanimação } \\
\text { cardiopulmonar }\end{array}$} \\
\hline Sim & $0(0,0)$ & $1(0,7)$ & $1(0,7)$ & \multirow{2}{*}{$0,600 * * *$} & $2(1,4)$ & $2(1,4)$ & $4(2,8)$ & \multirow{2}{*}{$1,000 * * *$} \\
\hline Não & $64(43,8)$ & $81(55,5)$ & $145(99,3)$ & & $34(23,6)$ & $106(73,6)$ & $140(97,2)$ & \\
\hline \multicolumn{9}{|c|}{$\begin{array}{l}\text { Uso de ventilação } \\
\text { mecânica }\end{array}$} \\
\hline Sim & $27(18,5)$ & $20(13,7)$ & $47(32,2)$ & \multirow{2}{*}{$0,022 *$} & $19(13,2)$ & $31(21,5)$ & $50(34,7)$ & \multirow{2}{*}{$0,008 *$} \\
\hline Não & $37(25,3)$ & $62(42,5)$ & $99(67,8)$ & & $17(11,8)$ & $77(53,5)$ & $94(65,3)$ & \\
\hline \multicolumn{9}{|c|}{$\begin{array}{l}\text { Reinternação } \\
\text { antes da alta } \\
\text { hospitalar }\end{array}$} \\
\hline Sim & $5(3,4)$ & $3(2,1)$ & $8(5,5)$ & \multirow{2}{*}{$0,900 * * *$} & $5(3,5)$ & $11(7,6)$ & $16(11,1)$ & \multirow{2}{*}{$0,800 * * *$} \\
\hline Não & $59(40,4)$ & $79(54,1)$ & $138(94,5)$ & & $31(21,5)$ & $97(67,4)$ & $128(88,9)$ & \\
\hline \multicolumn{9}{|l|}{$\begin{array}{l}\text { História } \\
\text { diagnóstica }\end{array}$} \\
\hline $\operatorname{Sim}$ & $51(34,9)$ & $10(6,8)$ & $61(41,7)$ & \multirow{2}{*}{$0,000^{*}$} & $27(18,8)$ & $46(31,9)$ & $73(50,7)$ & \multirow{2}{*}{$0,000 *$} \\
\hline Não & $13(8,9)$ & $72(49,3)$ & $85(58,3)$ & & $9(6,2)$ & $62(43,1)$ & $71(49,3)$ & \\
\hline \multicolumn{9}{|c|}{$\begin{array}{l}\text { Uso de tecnologia } \\
\text { em saúde }\end{array}$} \\
\hline $\operatorname{Sim}$ & $25(17,1)$ & $1(0,7)$ & $26(17,8)$ & \multirow{2}{*}{$0,000^{*}$} & $21(14,6)$ & $3(2,1)$ & $24(16,7)$ & \multirow{2}{*}{$0,000 * *$} \\
\hline Não & $39(26,7)$ & $81(55,5)$ & $120(82,2)$ & & $15(10,4)$ & $105(72,9)$ & $120(83,3)$ & \\
\hline \multicolumn{9}{|c|}{$\begin{array}{l}\text { Uso de medicação } \\
\text { contínua }\end{array}$} \\
\hline Sim & $29(19,9)$ & $3(2,1)$ & $32(21,9)$ & \multirow{2}{*}{$0,000 *$} & $32(22,2)$ & $23(16,0)$ & $55(38,2)$ & \multirow{2}{*}{$0,000 *$} \\
\hline Não & $35(24,0)$ & $79(54,1)$ & $114(78,1)$ & & $4(2,8)$ & $85(59,0)$ & $89(61,8)$ & \\
\hline
\end{tabular}

*Teste Qui-quadrado de Pearson. **Teste Qui-quadrado com correção de continuidade de Yates. ***Teste Exato de Fisher.

Fonte: Elaborado pelos autores (2020).

Na Tabela 4, observa-que uso de ventilação mecânica durante a internação, presença de história diagnóstica, dependência de tecnologia em saúde e uso de medicação contínua mostraram-se associadas à necessidade de cuidado contínuo e complexo. As classes medicamentosas que obtiveram maior destaque foram anticonvulsionantes $(1995=50 \%$; 1997=43,8\%; $2005=36,4 \% ; 2015=42,1 \% ; 2017=36,5 \%)$ e antidiabéticos $(2007=28,6 \%)$.

\section{Discussão}

A predominância do sexo masculino $(58,1 \%)$ nas internações em UTIP durante o período de 1995 a 2017 é corroborada por outros estudos realizados com esta população, pois retratam uma fragilidade na adaptação de crianças do sexo masculino quando comparadas às do sexo feminino (Santos et al., 2020; Araujo Filho et al., 2017; Arrué et al., 2014). Assim, observa-se 
que as diferenças biológicas podem resultar em uma maior suscetibilidade para o desenvolvimento de problemas de saúde e que potencializam as chances de se tornarem CRIANES (Arrué et al., 2014; Moreira et al., 2017).

Em relação à idade, a maioria $(45,4 \%)$ das CRIANES possuíam até um ano de idade e ao encontro deste achado, estudos evidenciam que a variável idade pode ser considerada como um fator de proteção para casos de reinternação. Isso, pode ser justificado pela observação de uma tendência de que crianças menores de um ano estão mais sujeitas à hospitalização quando comparadas com crianças com mais de um ano de idade, devido à imaturidade dos sistemas do organismo (Okido et al., 2016; Neuman et al., 2014).

Os participantes do estudo apresentaram muitos diagnósticos e de complexidades variadas, implicando em prolongadas e mais graves hospitalizações, frequente utilização de serviços de emergência e terapia intensiva. Essa realidade foi constatada a partir do quantitativo de crianças $(10,6 \%)$ que vivenciaram reinternações durante o período do estudo devido à condição de saúde apresentada, o que coincide com os achados de um estudo que caracterizou o público de CRIANES de acordo com suas condições clínicas, demandas de cuidados e situação sociodemográfica, em que apenas uma das 25 participantes não possuía histórico de internação pregressa (Santos et al., 2020). Com isso, percebe-se como os diagnósticos destas crianças são complexos e exigem uma vasta demanda de cuidados especiais.

A maioria das crianças possuía história de internações pregressas (87,9\%) e uso contínuo de medicamentos (66,1\%), reafirmando a necessidade de receber cuidados contínuos e complexos e que, além da possibilidade de acarretar importantes complicações para o crescimento e desenvolvimento, também demandam uma dedicação constante por parte da equipe de profissionais (Tavares et al., 2014).

Sobre as demandas de cuidados das CRIANES incluídas neste estudo, em sua totalidade necessitaram de cuidados habituais modificados, principalmente, relacionados à administração medicamentosa, ao risco de infecção, à alimentação, à higiene pessoal, entre outros. Ainda, no que tange à classificação de demandas, 40,4\% das CRIANES faziam uso de ventilação mecânica e 31,8\% possuíam a necessidade de utilizar alguma tecnologia em saúde.

Frente a isso, observa-se que crianças com diagnósticos crônicos de saúde possuem, geralmente, a necessidade de controle medicamentoso contínuo e, neste estudo, a classe mais utilizada por esta clientela correspondeu aos anticonvulsivantes. Estudo realizado com crianças com condição crônica egressas de uma Unidade de Terapia Intensiva Neonatal (UTIN) de Minas Gerais, também evidencia a grande utilização desta classe medicamentosa, sendo ultrapassada apenas pelos medicamentos procinéticos (Tavares et al., 2014). Este estudo também reafirma uma dependência de tecnologias em saúde para viabilizar uma melhor qualidade de vida a estas crianças tanto em ambiente hospitalar quanto em domicílio, ressaltando o uso de dispositivos voltados principalmente à alimentação e ao sistema respiratório (Tavares et al., 2014).

Os resultados dessa pesquisa demonstram que a maioria das CRIANES internam devido a comprometimentos do sistema respiratório $(41,9 \%)$, do sistema nervoso $(19,2 \%)$ e por doenças infecciosas e parasitárias $(17,2 \%)$, o que converge com outro estudo (Araujo Filho et al., 2017), que buscou identificar e caracterizar as crianças com necessidades especiais de saúde dentre os recém-nascidos egressos de uma UTIN, em que houve predomínio de internações por causas respiratórias e neurológicas, além de quadros de infecção durante a internação. Pesquisa realizada para avaliar o perfil de recém-nascidos internados em UTIN de hospitais públicos do sul do Brasil, relata que dentre os variados desfechos que levam à hospitalização, destacaram-se, também, as causas respiratórias e infecciosas (Silveira et al., 2020).

A infecção no período neonatal se configura em uma das principais causas de morbidade e mortalidade, uma vez que está diretamente relacionada à deficiência do sistema imunológico e à realização de procedimentos invasivos (Tames, 2017; Cardoso \& Schumacher, 2017). Em revisão sistemática da literatura desenvolvida para identificar eventos adversos e outros incidentes em UTIN, os achados demonstram que os casos de infecção são considerados eventos adversos evitáveis, pois são, majoritariamente, resultados advindos de falhas humanas em diferentes esferas do cuidado (Lanzillotti et al., 2015). Somado a 
isso, este estudo também evidencia um maior risco de infecção quando se trata de casos em que há necessidade do uso da ventilação mecânica, já que pode ocorrer extubação acidental, deslizes na extubação ou perda de cateteres, podendo, ainda, implicar em outras complicações (Lanzillotti et al., 2015).

A realidade das CRIANES que demandam de cuidados específicos para a sua sobrevivência, implica que profissionais de saúde aprendam além dos procedimentos técnicos que são realizados em ambiente hospitalar, também as orientações para os cuidadores que realizarão estes cuidados em domicílio após a desospitalização. Neste sentido, se em ambientes hospitalares preparados para fornecer cuidados contínuos e complexos há um desafio para os profissionais, a necessidade de aprender a lidar com a condição dessas CRIANES se configura em um desafio ainda maior para seus cuidadores. Isto resulta em modificações dos hábitos cotidianos familiares, quais sejam para atender às necessidades, por exemplo, de oxigenação, nutrição, conforto e bem-estar (Góes \& Cabral, 2017).

Com isso, tem-se o enfermeiro como profissional responsável por realizar educação em saúde, já que é de sua competência ensinar, explicar, incentivar a prática e observar a execução de cuidados pelo cuidador. Assim, a partir da mediação de saberes com fornecimento de informações e demonstração de como realizar o procedimento baseado nos princípios científicos, torna-se possível capacitar os cuidadores para que sejam parceiros deste cuidado durante a internação e, ainda, desenvolvam autonomia para realizar estes cuidados em domicílio, quando possível (Góes \& Cabral, 2017; Rodrigues et al., 2018).

\section{Conclusão}

O perfil da morbidade de crianças e adolescentes com cuidados contínuos e complexos, atendidos em uma UTIP de um hospital público localizado na Região Sul do Brasil, indicou as doenças do aparelho respiratório, doenças do sistema nervoso, doenças infecciosas e parasitárias, lesões, envenenamentos e algumas consequências de causas externas como as principais causas de internação nessa unidade. Houve predomínio em crianças de até um ano de idade, de etnia branca e do sexo masculino. História prévia de morbidades, uso de medicação contínua, de ventilação mecânica e de tecnologia em saúde mostraram-se condições associadas à necessidade de cuidados contínuos e complexos.

Evidenciou-se que o conhecimento de fatores que possam estar associados ao desenvolvimento de morbidades nesta clientela, pode possibilitar a sistematização de ações voltadas à redução da dependência de alguma tecnologia em saúde e de intercorrências no quadro clínico, além de melhor manejo do uso contínuo de medicações. Isso, pode potencializar uma diminuição do número de reinternações e da necessidade de cuidados contínuos e complexos, tanto no serviço de saúde quanto em domicílio, após a alta hospitalar.

Assim, com os resultados encontrados espera-se que profissionais, especialmente, os enfermeiros, consigam subsidiar o planejamento de cuidados direcionando para o perfil apresentado pela população em estudo, considerando suas necessidades individuais e coletivas. Cabe ao enfermeiro, também, a realização de educação em saúde com os cuidadores destas CRIANES para que possam estar orientados e instrumentalizados para desenvolver as demandas de cuidados que apresentam. Além disso, fazem-se necessários investimentos voltados à qualificação assistencial que proporcionem uma melhoria na qualidade de vida a estas CRIANES, como por exemplo, a capacitação dos profissionais de saúde da APS para atender a essa demanda.

Sugere-se o desenvolvimento de estudos futuros acerca da formação de recursos humanos para o cuidado desta clientela de modo multidisciplinar, a qualificação do parto e nascimento para reduzir os índices de intercorrências que ocasionem sequelas para a vida futura dessas crianças.

\section{Referências}

Araujo Filho A. C. A. et al. (2017). Aspectos epidemiológicos da mortalidade neonatal em capital do nordeste do Brasil. Rev Cuid Bucaramanga, 8(3): 17671776 . 
Arrué, A. M. et al. (2016). Tradução e adaptação do Children With Special Health Care Needs Screener para o português. Cad Saúde Pública (Online), 32(6): e00130215

Arrué, A. M. et al. (2014). Crianças com necessidades especiais de saúde egressas de terapia intensiva neonatal. Evidentia, 11(45).

Brasil. (1990). Lei n 8069, de 13 de julho de 1990. Dispõe sobre o Estatuto da Criança e do Adolescente e dá outras providências. Diário Oficial da República Federativa do Brasil. https://www.gov.br/mdh/pt-br/assuntos/noticias/2021/julho/trinta-e-um-anos-do-estatuto-da-crianca-e-do-adolescente-confira-as-novasacoes-para-fortalecer-o-eca/ECA2021_Digital.pdf

Brasil. (2010). Ministério da Saúde. Agência Nacional de Vigilância Sanitária. Resolução no 7, de 24 de fevereiro de 2010 . Dispõe sobre os requisitos mínimos para funcionamento de Unidades de Terapia Intensiva e datras a https://bvsms.saude.gov.br/bvs/saudelegis/anvisa/2010/res0007_24_02_2010.html.

Cardoso, D. J. S. \& Schumacher, B. (2017). Epidemiological characteristics of neonatal admissions in a public maternity. Rev Enferm UFP, 6(4): 28-32.

Cohen, E. et al. (2011). Children with medical complexity: an emerging population for clinical and research initiatives. Pediatrics, 127(3): 529-38.

Cruz, C. T. et al. (2017). Care to children requiring continuous and complex assistance: nursing perception. REME Rev Min Enferm, 21 : e-1005.

Góes, F. G. B. \& Cabral, I. E. (2017). Discourses on discharge care for children with special healthcare needs. Rev Bras Enferm, 70(1): 154-61.

Hockenberry, M. J. \& Wilson, D. (2014). Wong: fundamentos de enfermagem pediátrica. $9^{\text {a }}$ ed. Rio de Janeiro: Elsevier.

Lanzillotti, L. S. et al. (2015). Eventos adversos e outros incidentes na unidade de terapia intensiva neonatal. Cien Saude Colet, 20(3): 937-946.

Mcpherson, M. G. et al. (1998). A new definition of children with special health care needs. Pediatrics, 102(1): 137-139.

Moreira, K. F. A. et al. (2017). Perfil e evitabilidade de óbito neonatal em um município da Amazônia Legal. Cogitare Enferm, 22(2): e48950.

Neuman, M. I. et al. (2014). Readmissions Among Children Previously Hospitalized With Pneumonia. Shah Pediatrics, 134(1): 100-109.

Neves, E. T. \& Cabral, I. E. (2009). Cuidar de crianças com necessidades especiais de saúde: desafios para as famílias e enfermagem pediátrica. Rev Eletr Enf, $11(3)$.

Neves, E. T. et al. (2019). Acesso de crianças com necessidades especiais de saúde à rede de atenção. Revista Bras Enferm, 72(Suppl. 3): 65-71.

Okido, A. C. C., Pina, J. C. \& Lima, R. A. G. (2016). Fatores associados às internações não eletivas em crianças dependentes de tecnologia. Rev. esc. enferm. USP, 50(1): 29-35.

Polit, D. F. \& Beck, C. T. Fundamentos de pesquisa em enfermagem. $7^{\text {a }}$ ed. Porto Alegre; Editora Artmed, 2011.

Rodrigues, D. Z., Ferreira, F. Y. \& Okido, A. C. (2018). Sobrecarga do cuidador familiar de crianças com necessidades especiais de saúde. Rev eletrônica enferm, 20.

Santos, R. P. et al. (2020). Perfil de crianças com necessidades especiais de saúde e seus cuidadores em um hospital de ensino. Cienc Cuid Saúde, 19 : e46724.

Silva, R. et al. (2017). A longitudinalidade no cuidado à saúde da criança no contexto da atenção primária. Revista de Enfermagem UFPE on line, 11(5): 19091917.

Silveira, A. \& Neves, E. T. (2019). Cotidiano de cuidado de adolescentes com necessidades especiais de atenção à saúde. Acta Paul Enferm, 32 (3): 327-333.

Silveira, T. B. et al. (2020). Perfil epidemiológico de recém-nascidos internados em Unidades de Terapia Intensiva Neonatal em hospitais universitários no extremo Sul do Brasil. Vittalle - Revista de Ciências da Saúde, 32(2): 46-54.

Simon, T. D. et al. (2010). Children with complex chronic conditions in inpatient hospital settings in the United States. Pediatrics, 126(4): 647-55.

Sulino, M. C. et al. (2021). Crianças e adolescentes com necessidades especiais de saúde: (des)continuidade do cuidado. Texto contexto - enferm, 30 : e20190363.

Tames, R. N. (2017). Enfermagem na UTI neonatal: assistência ao recém-nascido de alto risco. Rio de Janeiro: $6^{\mathrm{a}}$ Ed. Guanabara Koogan.

Tavares, T. S. et al. (2014). Caracterização do perfil das crianças egressas de unidade neonatal com condição crônica. R. Enferm. Cent. O. Min, 3(4): 1322-1335. 